\title{
Hormetic Effects of Trifloxystrobin on Aggressiveness of Sclerotinia sclerotiorum
}

Ya-Li Di, Meng-Long Cong, Ran Zhang, and Fu-Xing Zhu, College of Plant Science and Technology, Huazhong Agricultural University, Wuhan, 430070, China

\begin{abstract}
Di, Y.-L., Cong, M.-L., Zhang, R., and Zhu, F.-X. 2016. Hormetic effects of trifloxystrobin on aggressiveness of Sclerotinia sclerotiorum. Plant Dis. 100:2113-2118.

Sclerotinia sclerotiorum is a devastating ascomycete plant pathogen with an extremely wide host range. Fungicides are still the mainstay for control of this pathogen, and stimulations to mycelial growth and aggressiveness by subtoxic doses of fungicides carbendazim and dimethachlon have been reported. The present study assessed hormetic effects of the quinone outside inhibitor $(\mathrm{QoI})$ fungicide trifloxystrobin on aggressiveness of $S$. sclerotiorum. Trifloxystrobin at 0.0001, 0.0005, and $0.001 \mu \mathrm{g} / \mathrm{ml}$ exerted significant stimulatory effects on aggressiveness to potted rapeseed plants, and the highest percent stimulation were 20.5 and $24.2 \%$ for isolates HB15 and SX11, respectively. At $18 \mathrm{~h}$ postinoculation (HPI), initial necrotic lesions were visible to the naked eye on leaves treated with trifloxystrobin, whereas no obvious disease symptoms were discerned for the nontreated control. At 24, 36, and $48 \mathrm{HPI}$, aggressiveness stimulation was more obvious than at $18 \mathrm{HPI}$. Scanning electron microscopic observations demonstrated that no mycelia were detected on the

nontreated leaves at $4 \mathrm{HPI}$; by contrast, mycelia were observed on leaves treated with trifloxystrobin at $0.0001 \mu \mathrm{g} / \mathrm{ml}$. At 8 and $12 \mathrm{HPI}$, there were more mycelia and infecting hyphae on the treated leaves than on the nontreated control. These results indicated that fungal stimulation had occurred in the first 4 and $8 \mathrm{HPI}$, suggesting that direct stimulation was likely to be the underlying mechanism for hormetic actions of trifloxystrobin. Pretreatment with trifloxystrobin did not significantly affect subsequent mycelial growth on PDA or aggressiveness to detached rapeseed leaves in the absence of trifloxystrobin. However, in the presence of trifloxystrobin, mycelial growth and aggressiveness were significantly $(P<0.05)$ greater for the pretreatment with trifloxystrobin at 0.003 and $0.03 \mu \mathrm{g} / \mathrm{ml}$ compared with the nonpretreatment control, indicating that a prior exposure to the fungicide may undermine its subsequent effectiveness. These studies will raise our awareness of fungicide hormesis and have important implications for judicious application of fungicides.
\end{abstract}

Sclerotinia sclerotiorum (Lib.) de Bary, the causal agent of Sclerotinia stem and head rot diseases on many economically important crops, is a necrotrophic fungal pathogen with a broad host range. This pathogen can infect at least 408 species of plants, most of which are dicotyledons (Boland and Hall 1994; Bolton et al. 2006). Diseases caused by $S$. sclerotiorum usually lead to substantial yield losses in crops such as rapeseed, sunflower, soybean, and dry bean (McCreary et al. 2016; Wang et al. 2014). According to the United States Department of Agriculture (USDA 2012), annual economic loss attributed to $S$. sclerotiorum in only five crops (i.e., soybean, sunflower, dry editable bean, canola, and pulse) is about $\$ 482$ million, on average, in America. Fungicides have been the major and most effective measure for control of this cosmopolitan pathogen. The benzimidazole fungicide carbendazim has been widely employed in China to control S. sclerotiorum in the last four decades. High levels of carbendazim resistance began to emerge in the middle 1990s and, thus far, still remain at relatively high frequencies in eastern China (Pan et al. 1997; Wang et al. 2014; Xu et al. 2015; Zhu et al. 2016). Due to prevalence of carbendazim resistance, the dicarboximide fungicide dimethachlone was recommended in Jiangsu Province of eastern China as a substitution fungicide for management of Sclerotinia stem rot disease on rapeseed. Unsurprisingly, dimethachlone resistance in $S$. sclerotiorum has been detected in recent years in major rapeseedand soybean-growing regions of China (Ma et al. 2009; Wang et al. 2014; Zhou et al. 2014b).

While monitoring resistance to carbendazim and dimethachlone, we observed that low doses of each of the two fungicides increased mycelial growth rate and aggressiveness of $S$. sclerotiorum, especially in fungicide-resistant isolates (Di et al. 2015; Zhou et al. 2014a). Dimethachlone at concentrations from 1 to $5 \mu \mathrm{g} / \mathrm{ml}$ displayed

Corresponding author: F.-X. Zhu; E-mail: zhufuxing@mail.hzau.edu.cn

Accepted for publication 6 June 2016.

http://dx.doi.org/10.1094/PDIS-03-16-0403-RE

(C) 2016 The American Phytopathological Society stimulatory effects on S. sclerotiorum aggressiveness on rapeseed plants (Zhou et al. 2014a). Field efficacy trials showed that dimethachlone at $300 \mu \mathrm{g} / \mathrm{ml}$ significantly enhanced aggressiveness of a dimethachloneresistant isolate on rapeseed plants (Firoz et al. 2016). In the laboratory, carbendazim at 0.2 to $5 \mu \mathrm{g} / \mathrm{ml}$ enhanced aggressiveness of carbendazimresistant isolates by more than $30 \%$ (Di et al. 2015). A further study indicated that direct stimulation rather than overcompensation to the disruption of homeostasis was likely to be the underlying mechanism for aggressiveness stimulation (Di et al. 2016a). These stimulatory effects of low doses of dimethachlone and carbendazim on aggressiveness of S. sclerotiorum conform to the concept of hormesis. Hormesis is a toxicological concept of dose-response relationships characterized by lowdose stimulation and high-dose inhibition; or, more strictly speaking, hormesis refers to a reversal of response between low and high doses of a stressor (Calabrese 2015a; Hoffmann 2009). Hormesis is considerably generalizable. Chemical hormesis has been observed in a wide range of biological models, over numerous and diverse measurement endpoints, across a variety of chemical classes, and independent of underlying mechanisms (Calabrese 2013, 2015a). Calabrese and Blain (2011) established a hormesis database and found that approximately 9,000 dose responses in the published literature satisfied a priori entry and evaluative criteria. Plenty of fungicide hormesis cases have been reported, such as stimulatory effects of thiabendazole on Penicillium expansum (Baraldi et al. 2003); propamocarb on Lyophyllum palustre, Pythium aphanidermatum, P. irregulare, and P. ultimum (Landry et al. 2011; Moorman and Kim 2004); metalaxyl on Phytophthora infestans (Zhang et al. 1997); and mefenoxam on Pythium aphanidermatum (Garzón et al. 2011).

Hormetic effects of fungicide on phytopathogens, especially stimulation of mycelial growth and aggressiveness, have important implications for fungicide applications. Under field conditions, a portion of pathogens or isolates are likely to be exposed to low doses of a fungicide due to evaporation, drift, or uneven distribution of the active ingredient (Flores and Garzón 2013). Recommended application rates on fungicide labels are, in fact, relatively low or subtoxic to fungicide-resistant isolates. Subtoxic doses of a fungicide are likely to have moderate stimulatory effects on aggressiveness of pathogens, potentially resulting in more severe plant diseases. In rapeseed fields 
with a high frequency of carbendazim resistance, Sclerotinia stem rot disease may occasionally become aggravated after application of carbendazim (Di et al. 2015). To control a plant disease successfully, a fungicide is usually applied two or more times in one growing season. In theory, a prior exposure of pathogens to a fungicide may potentially affect the efficacy of subsequent application of the same or other fungicide through a mechanism known as preconditioning. Preconditioning refers to a phenomenon in which a prior exposure to relatively low doses of a toxic agent or stress will reduce toxicity of subsequent exposure to relatively high doses of the same, a related, or even a nonrelated toxic agent or stressor. Preconditioning has been investigated and reported mainly in biomedical sciences, and has been regarded as a specific type of hormesis (Calabrese 2016a,b). Assessment and quantification of hormetic effects of fungicides on target pathogens are of great importance for avoiding the adverse effects of fungicide hormesis.

The quinone outside inhibitor (QoI) fungicides are highly effective to a wide variety of fungal pathogens, and have been extensively used worldwide in agriculture as the most important fungicide class with respect to market share and sales (Bartlett et al. 2002; Wise et al. 2008). Fungicidal activity and efficacy of the QoI fungicides azoxystrobin (Duan et al. 2012), benzothiostrobin (Xu et al. 2014), pyraclostrobin (Liang et al. 2015), and trifloxystrobin (Di et al. 2016b) against $S$. sclerotiorum have been reported, although no QoI fungicides have been formally registered in China for control of S. sclerotiorum (China Pesticide Information Network, http://www.chinapesticide. gov.cn). Trifloxystrobin, a member of the QoI class, has translaminar and redistribution properties in plants and can provide excellent protective and curative efficacy against a broad range of plant pathogens (Margot et al. 1998). Our preliminary studies showed that trifloxystrobin was highly efficacious and had a potential to be registered and used as a substitute fungicide in management of S. sclerotiorum (Di et al. 2016b). Studies on hormetic effects of trifloxystrobin are of practical significance for scientific application of this fungicide. The specific objectives of the present study were to (i) quantify stimulatory effects of low doses of trifloxystrobin on aggressiveness of S. sclerotiorum, (ii) explore the underlying hormetic mechanisms by assessing temporal profile of the stimulatory actions, and (iii) determine the effects of a prior exposure to trifloxystrobin on later mycelial growth and aggressiveness of $S$. sclerotiorum in the presence and absence of this fungicide.

\section{Materials and Methods}

Isolates of $\boldsymbol{S}$. sclerotiorum. Two field isolates of S. sclerotiorum (HB15 and SX11 from Hubei and Anhui provinces, respectively) were collected in 2013 from diseased rapeseed plants. Mature sclerotia were stored at $4{ }^{\circ} \mathrm{C}$ in a refrigerator before being cultured on potato dextrose agar (PDA) media.

Fungicide. Technical-grade trifloxystrobin (97\% active ingredient; Hubei Jianyuan Chemical Co. Ltd.) was dissolved in $100 \%$ pure acetone to produce a stock solution of $1,000 \mu \mathrm{g} / \mathrm{ml}$. The stock solution was stored at $4{ }^{\circ} \mathrm{C}$ in a refrigerator.

Stimulatory effects of low doses of trifloxystrobin on aggressiveness to detached leaves of rapeseed plants. The procedure was according to Di et al. (2015), with slight modifications. Trifloxystrobin stock solution was diluted first with $100 \%$ pure acetone, then with $0.1 \%$ Triton-100 in water to final concentrations of 0.00005 , $0.0001,0.0005,0.001,0.005$, and $0.05 \mu \mathrm{g} / \mathrm{ml}$. Acetone diluted in $0.1 \%$ Triton- 100 at a final concentration of $0.05 \%$ ( $\mathrm{vol} / \mathrm{vol}$ ) was used as the nontreated control. Trifloxystrobin dilutions were sprayed with a hand-held sprayer ( $800 \mathrm{ml}$ in volume; Xinmeir Co. Ltd.). Leaves of 8 to $11 \mathrm{~cm}$ in diameter were detached from 4-week-old rapeseed plants growing in the greenhouse at $23^{\circ} \mathrm{C}$. The detached leaves were rinsed with sterile water three times, air dried for $1 \mathrm{~h}$, sprayed with trifloxystrobin dilutions, and transferred to 15 -cm-diameter petri dishes lined with wetted filter paper to maintain high humidity. The treated leaves were air dried for $1 \mathrm{~h}$, then inoculated on the adaxial surface with inverted mycelial plugs cut from margins of 2-day-old S. sclerotiorum colonies. The inoculated leaves in lidded petri dishes were incubated in a growth chamber at $23^{\circ} \mathrm{C}$, with relative humidity maintained at about
$85 \%$. Lesion diameters were measured twice at right angles after $48 \mathrm{~h}$ of incubation. The experiment was performed in triplicate and repeated independently four times.

Stimulatory effects of low doses of trifloxystrobin on aggressiveness to potted rapeseed plants. Rapeseed plants, about 4-weekold, growing in 20-cm-diameter pots containing composite mixtures of peat and soil (weight ratio $=1: 1$ ), were sprayed with trifloxystrobin dilutions at concentrations of $0.0001,0.0005,0.001,0.005,0.01,0.05$, 0.1 and $0.5 \mu \mathrm{g} / \mathrm{ml}$ until runoff for both isolates, and a concentration of $0.00005 \mu \mathrm{g} / \mathrm{ml}$ was added for isolate HB15 because of the high percent stimulation at $0.0001 \mu \mathrm{g} / \mathrm{ml}$. After air drying for $1 \mathrm{~h}$, leaves were inoculated on the adaxial surface with inverted mycelial plugs (one plug per leaf, two leaves per plant). The inoculated plants were incubated in a growth chamber at $23^{\circ} \mathrm{C}$, with relative humidity maintained at about $85 \%$. Lesion diameters were measured twice at right angles after $48 \mathrm{~h}$ of incubation. The experiment was performed in triplicate and repeated independently four times.

Temporal profile of aggressiveness stimulation. Determination of the temporal profile of aggressiveness stimulation was according to Di et al. (2016a), with minor modifications. Leaves were detached, rinsed, air dried, sprayed with trifloxystrobin, inoculated, and incubated as described above. The concentrations of trifloxystrobin spayed were 0.0001 and $0.0005 \mu \mathrm{g} / \mathrm{ml}$. Leaves sprayed with acetone at $0.05 \%(\mathrm{vol} / \mathrm{vol})$ in $0.1 \%$ Triton- 100 in water were regarded as the nontreated control. Disease symptoms were photographed with a digital camera (Canon Powershot G12) at 8, 12, 18, 24, 36, and $48 \mathrm{~h}$ postinoculation (HPI). The experiment was performed in triplicate and repeated independently twice.

Electron microscopic observations of $S$. sclerotiorum infection process. Leaves were selected, sprayed with trifloxystrobin dilutions, inoculated, and incubated as described above. The trifloxystrobin treatment rate was $0.0001 \mu \mathrm{g} / \mathrm{ml}$, and leaves sprayed with acetone in $0.1 \%$ Trion- 100 were used as the nontreated control. The inoculated leaves were sampled at 4, 8, and 12 HPI. Sample preparations and observations with scanning electron microscopy (SEM) were performed according to Zhang et al. (2010). At sampling time points, mycelial plugs were removed and the inoculated segments of leaves were cut into small pieces ( 3 by $3 \mathrm{~mm}$ ). The samples were fixed in $2.5 \%$ (wt/vol) glutaraldehyde solution in sodium phosphate buffer $(0.05 \mathrm{M}, \mathrm{pH} 7.0)$ at $4^{\circ} \mathrm{C}$ for $24 \mathrm{~h}$, washed three times in $0.05 \mathrm{M}$ sodium phosphate buffer for $10 \mathrm{~min}$ each time, then dehydrated in a graded ethanol series. After critical point drying and gold-coating in a sputter coater (JFC-1600; NTC), the leaf specimens were examined with SEM (JSM-6390/LV; NTC). The experiment was conducted in triplicate.

Effect of pretreatment with trifloxystrobin on mycelial growth and aggressiveness of $\boldsymbol{S}$. sclerotiorum in the presence and absence of this fungicide. Mycelial plugs of isolate SX11 were placed on trifloxystrobin-amended PDA and grown at $23^{\circ} \mathrm{C}$ for $48 \mathrm{~h}$. The final concentrations of trifloxystrobin in PDA were 0 (an equal volume of acetone instead of trifloxystrobin solution was added), 0.003, 0.03, and $0.1 \mu \mathrm{g} / \mathrm{ml}$. Mycelial plugs from the margins of growing colonies on PDA amended with trifloxystrobin (as the pretreatment) were placed upside down on the centers of PDA plates amended with trifloxystrobin at $0.05 \mu \mathrm{g} / \mathrm{ml}$ or inoculated on detached leaves sprayed with trifloxystrobin at $1.5 \mu \mathrm{g} / \mathrm{ml}$. PDA amended with only acetone and leaves sprayed with $0.1 \%$ Triton- 100 in water were regarded as the nontreated control. After $48 \mathrm{~h}$ of incubation at $23^{\circ} \mathrm{C}$, diameters of mycelial colonies and disease lesions were measured twice at right angles. The experiments were repeated independently three times and there were 12 replicates in total for each treatment.

Data analysis. Percent stimulation was calculated by the following formula: percent stimulation $(\%)=[($ lesion diameter of the treated lesion diameter of the control)/lesion diameter of the control] $\times 100 \%$.

Percent stimulation was arcsine square-root transformed before subsequent statistical analysis. Dunnett's test $(\alpha=0.05)$ following one-way analysis of variance in SPSS (Statistical Product and Service Solutions, ver. 21.0; SPSS Inc.) was employed to evaluate the significance of percent stimulation compared with the nontreated control. The experimental unit in the pot experiment was defined 
as one rapeseed plant growing in one pot, with the arithmetic mean of lesion diameters as the response. Graphs were created with GraphPad Prism (ver. 5.01; GraphPad Software, Inc.) and Microsoft Excel (ver. 2010; Microsoft Corporation).

\section{Results}

Stimulatory effects of trifloxystrobin on aggressiveness to detached leaves of rapeseed plants. Statistically significant $(P<0.05)$ aggressiveness stimulation was detected for trifloxystrobin at 0.0001 and $0.0005 \mu \mathrm{g} / \mathrm{ml}$ to both isolates tested (Fig. 1). The highest percent stimulation was $18.9 \%$ for isolate $\mathrm{HB} 15$ and $13.9 \%$ for isolate SX1, both occurring at $0.0001 \mu \mathrm{g} / \mathrm{ml}$. Trifloxystrobin at $0.05 \mu \mathrm{g} / \mathrm{ml}$ displayed inhibitory rather than stimulatory effects on aggressiveness of S. sclerotiorum.

Stimulatory effects on aggressiveness of $S$. sclerotiorum to potted rapeseed plants. Trifloxystrobin at $0.0001,0.0005$, and 0.001 $\mu \mathrm{g} / \mathrm{ml}$ elicited significant $(P<0.05)$ aggressiveness stimulation of the two isolates tested (Fig. 2). For isolate HB15, the highest percent stimulation was $20.5 \%$, occurring with trifloxystrobin at $0.001 \mu \mathrm{g} / \mathrm{ml}$. For isolate $\mathrm{SX} 11$, the highest percent stimulation was $24.2 \%$, occurring at $0.0005 \mu \mathrm{g} / \mathrm{ml}$.

Temporal profile of aggressiveness stimulation of $S$. sclerotiorum. At 8 and 12 HPI, no necrotic lesions could be discerned by the naked eye for all the treatments (Fig. 3). At 18 HPI, initial necrotic lesions were visible only for leaves treated with trifloxystrobin at $0.0005 \mu \mathrm{g} / \mathrm{ml}$, whereas no necrotic lesions were detected for the nontreated control. From 24 to 48 HPI, disease lesions on leaves treated with trifloxystrobin
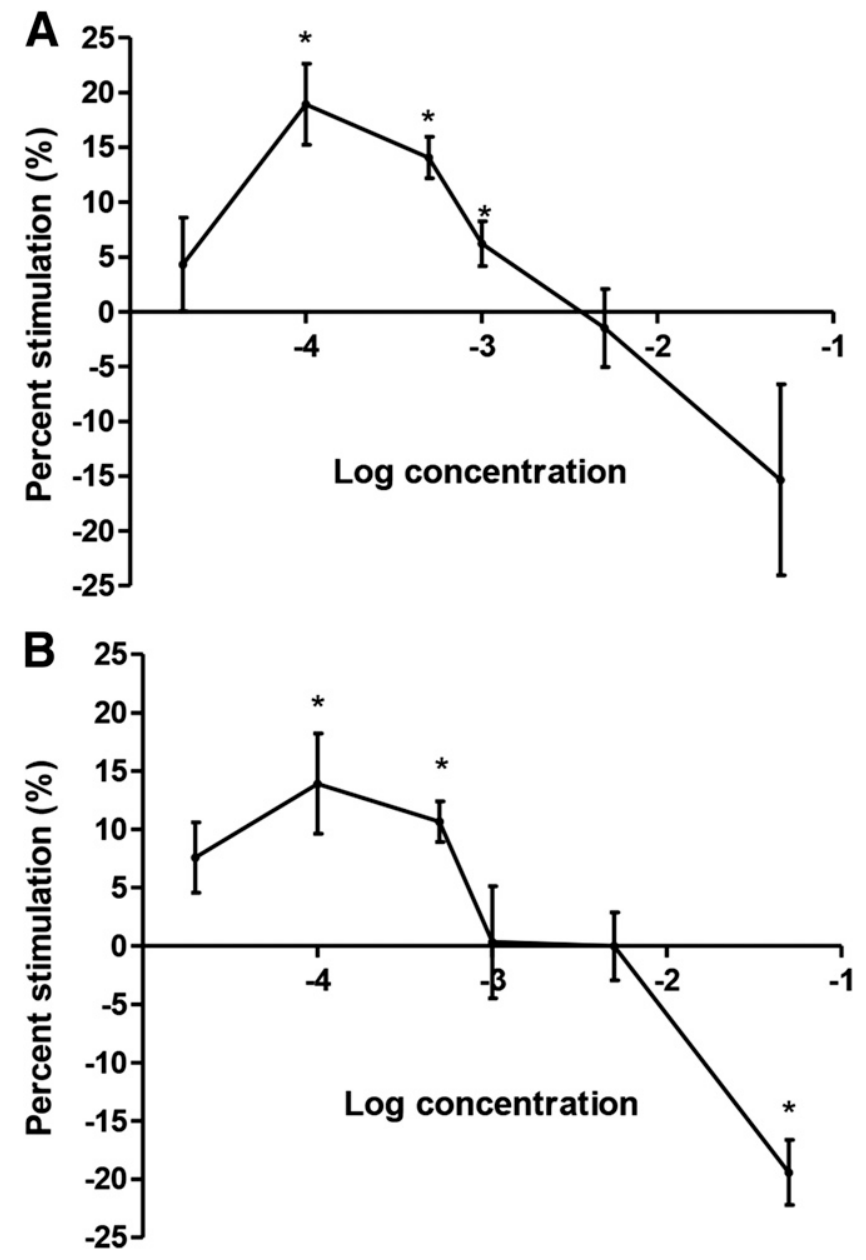

Fig. 1. Aggressiveness stimulation by trifloxystrobin to Sclerotinia sclerotiorum isolates A, HB15 and B, SX11 on detached leaves of oilseed rape plants. Concentrations of trifloxystrobin from left to right on the $x$-axis are $0.00005,0.0001,0.0005,0.001$, 0.005 , and $0.05 \mu \mathrm{g} / \mathrm{ml}$. Error bars denote standard error of the mean. Asterisks denote significant differences from the control. Data are mean values of 12 replicates. at 0.0001 and $0.0005 \mu \mathrm{g} / \mathrm{ml}$ were progressively larger than the nontreated control.

Electron microscopic observations of $S$. sclerotiorum infection process. Observations with SEM demonstrated that, at $4 \mathrm{HPI}$, no infection structures such as infection cushions or even mycelia could be detected for the nontreated control. However, for leaves treated with trifloxystrobin at $0.0001 \mu \mathrm{g} / \mathrm{ml}$, hyphae penetrating into the epidermis of leaves could be observed (Fig. 4). At 8 and 12 HPI, hyphae grew faster and there were more mycelia adhering to leaves for treatment with trifloxystrobin compared with the nontreated control.

Effects of pretreatment with trifloxystrobin on mycelial growth and aggressiveness of $S$. sclerotiorum in the presence and absence of this fungicide. After mycelia were grown on trifloxystrobinamended PDA at $23^{\circ} \mathrm{C}$ for $48 \mathrm{~h}$ (as pretreatment), percent inhibition of mycelial growth was $22.0,29.4$, and $60.5 \%$ for trifloxystrobin at $0.003,0.03$, and $0.1 \mu \mathrm{g} / \mathrm{ml}$, respectively. Mycelial plugs from the pretreated colonies were placed on PDA media supplemented with and without trifloxystrobin, and on detached leaves treated with and without trifloxystrobin. The subsequent mycelial growth rates on PDA without trifloxystrobin were similar among different pretreatments (Fig. 5). However, mycelial growth rates for the pretreated mycelial plugs on PDA amended with $0.05 \mu \mathrm{g} / \mathrm{ml}$ trifloxystrobin were significantly higher $(P<0.05)$ than the nonpretreated control. Lesion progress on detached rapeseed leaves showed a similar trend. There were no significant differences in lesion diameter on the nontreated leaves among different pretreatments. However, on leaves treated with trifloxystrobin at $1.5 \mu \mathrm{g} / \mathrm{ml}$, lesion diameters for mycelial plugs pretreated
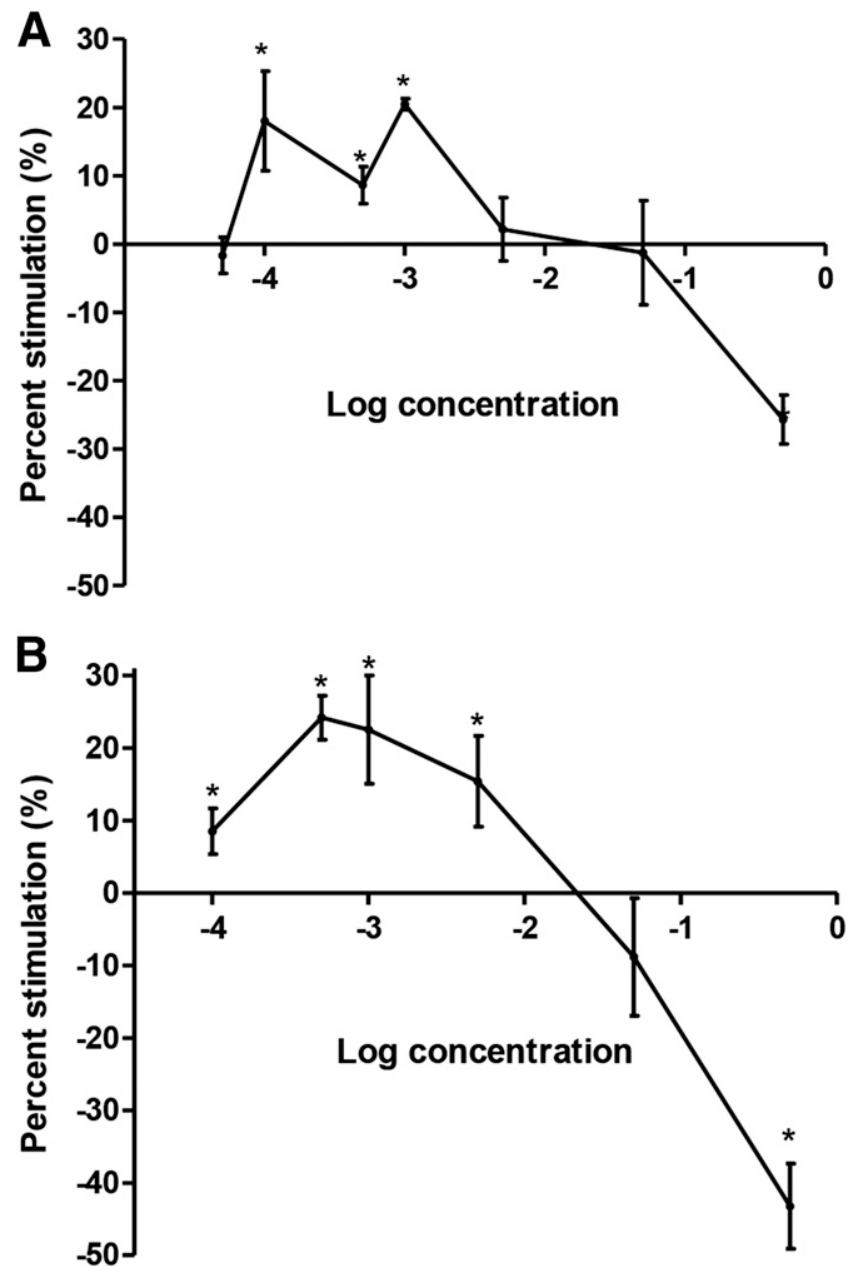

Fig. 2. Aggressiveness stimulation of Sclerotinia sclerotiorum by trifloxystrobin treatment for isolates A, HB15 and B, SX11 on potted oilseed rape plants. Error bars denote standard error of the mean. Asterisks denote significant differences from the control. Data are mean values of 12 replicates. 
with trifloxystrobin at 0.003 and $0.03 \mu \mathrm{g} / \mathrm{ml}$ were significantly greater than the nonpretreated control.

\section{Discussion}

The present study demonstrated that trifloxystrobin at 0.0001 and $0.0005 \mu \mathrm{g} / \mathrm{ml}$ elicited statistically significant stimulation of aggressiveness of $S$. sclerotiorum on rapeseed plants. The maximum stimulation magnitude was $24.2 \%$, occurring on potted rapeseed plants treated with trifloxystrobin at $0.0005 \mu \mathrm{g} / \mathrm{ml}$. The initial signs of stimulation could be observed at 18 HPI with the naked eye, and as early as 4 HPI with SEM on rapeseed plants. Pretreating mycelia with trifloxystrobin at 0.003 and $0.03 \mu \mathrm{g} / \mathrm{ml}$ increased mycelial growth and aggressiveness of $S$. sclerotiorum in the presence of this fungicide compared with the nonpretreatment control.

The dose-response curves in the present study were, in general, consistent with the concept of hormesis. Maximum stimulation magnitudes of hormesis are often approximately 30 to $60 \%$ greater than the control, and hormetic dose zones are usually 10- to 20 -fold immediately following the level of no observed adverse effect (Calabrese 2015a). The present study showed that the maximum aggressiveness stimulation was $24.2 \%$ and the stimulation dose zone for trifloxystrobin was about 10- to 15-fold. These features indicated that the biphasic dose responses of $S$. sclerotiorum to trifloxystrobin conformed to the concept of hormesis. Hormesis is highly generalizable, independent of biological model, inducing agent, endpoint, and mechanism (Calabrese 2015a; Calabrese and Blain 2011). In addition to trifloxystrobin, carbendazim and dimethachlone also exhibited hormetic effects on mycelial growth and aggressiveness of S. sclerotiorum (Di et al. 2015;

12 HPI
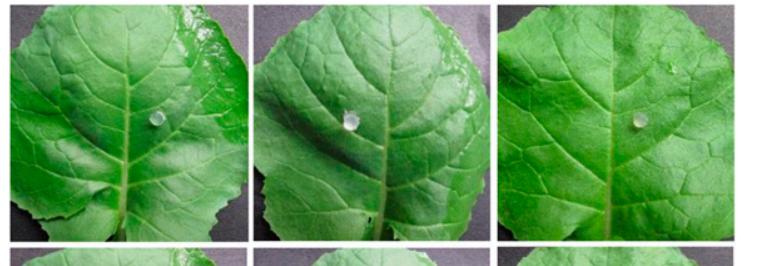

18 HPI
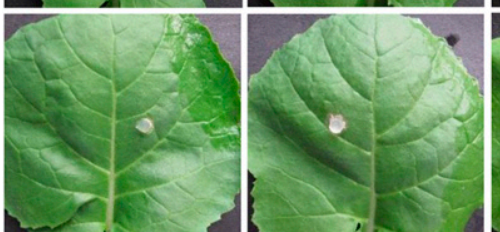

24 HPI
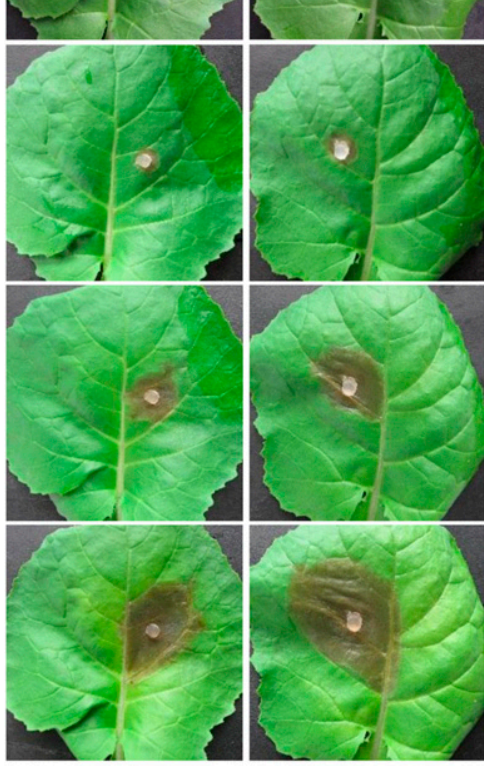

$0.0001 \mu \mathrm{g} / \mathrm{ml}$

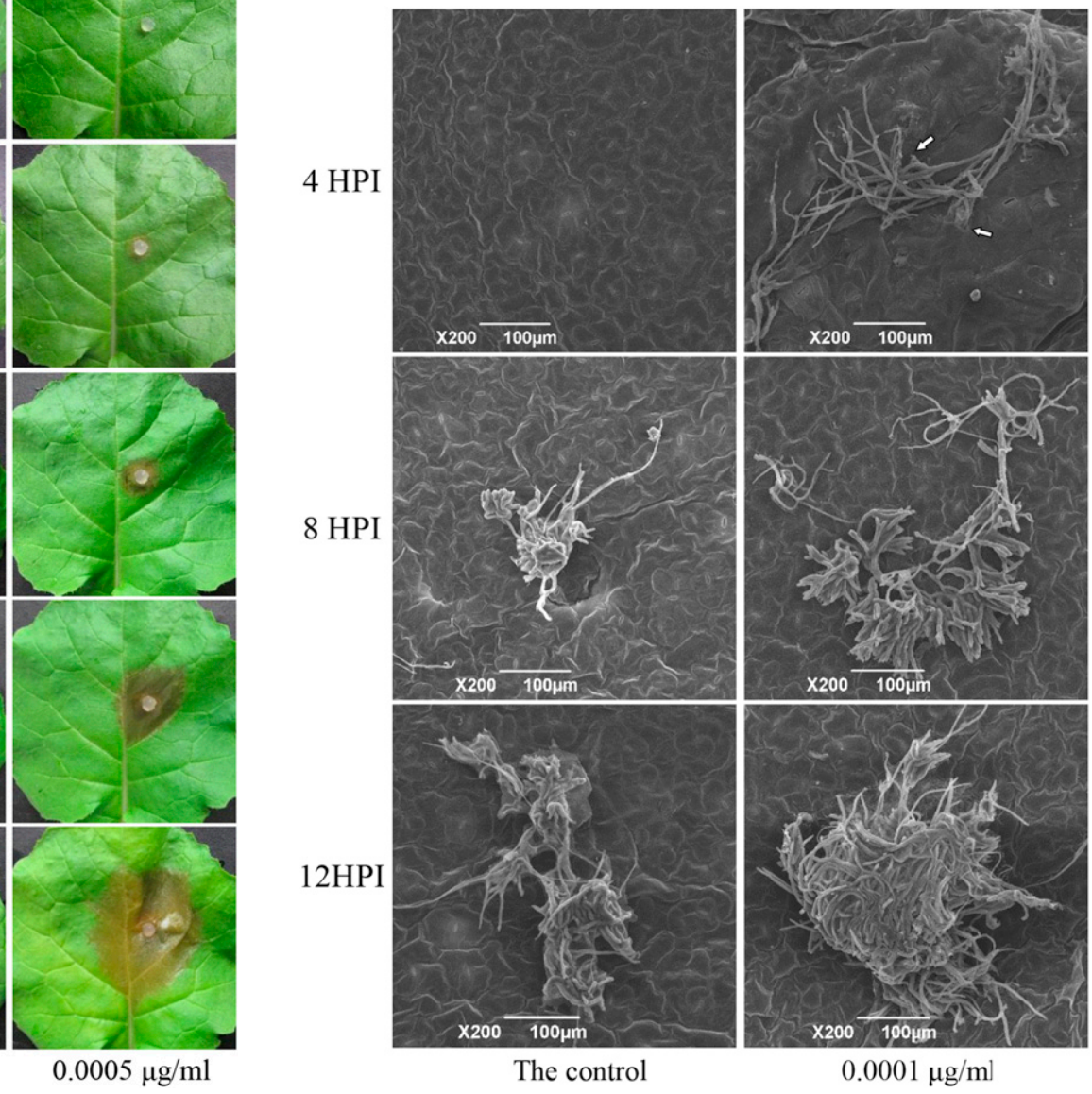

36HPI

48 HPI
The control

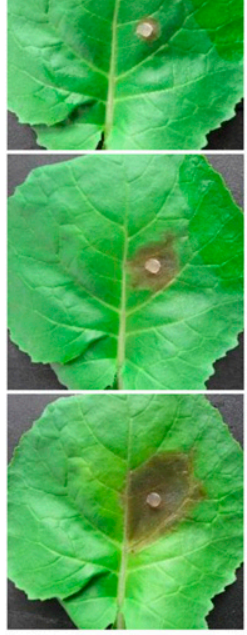

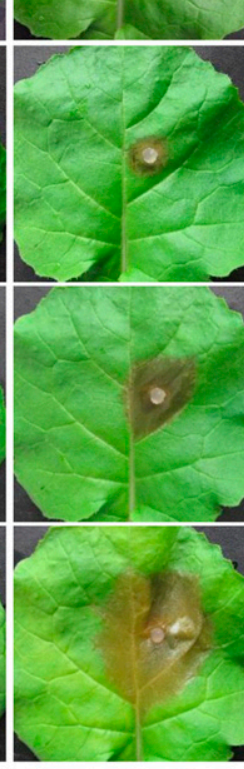

$0.0005 \mu \mathrm{g} / \mathrm{ml}$
Fig. 3. Temporal profile of aggressiveness stimulation of Sclerotinia sclerotiorum by trifloxystrobin for isolate SX11 on leaves of oilseed rape plants. Leaves treated with $0.1 \%$ Triton-100 in water in the first column were used as the nontreated control. $\mathrm{HPI}=$ hours postinoculation.

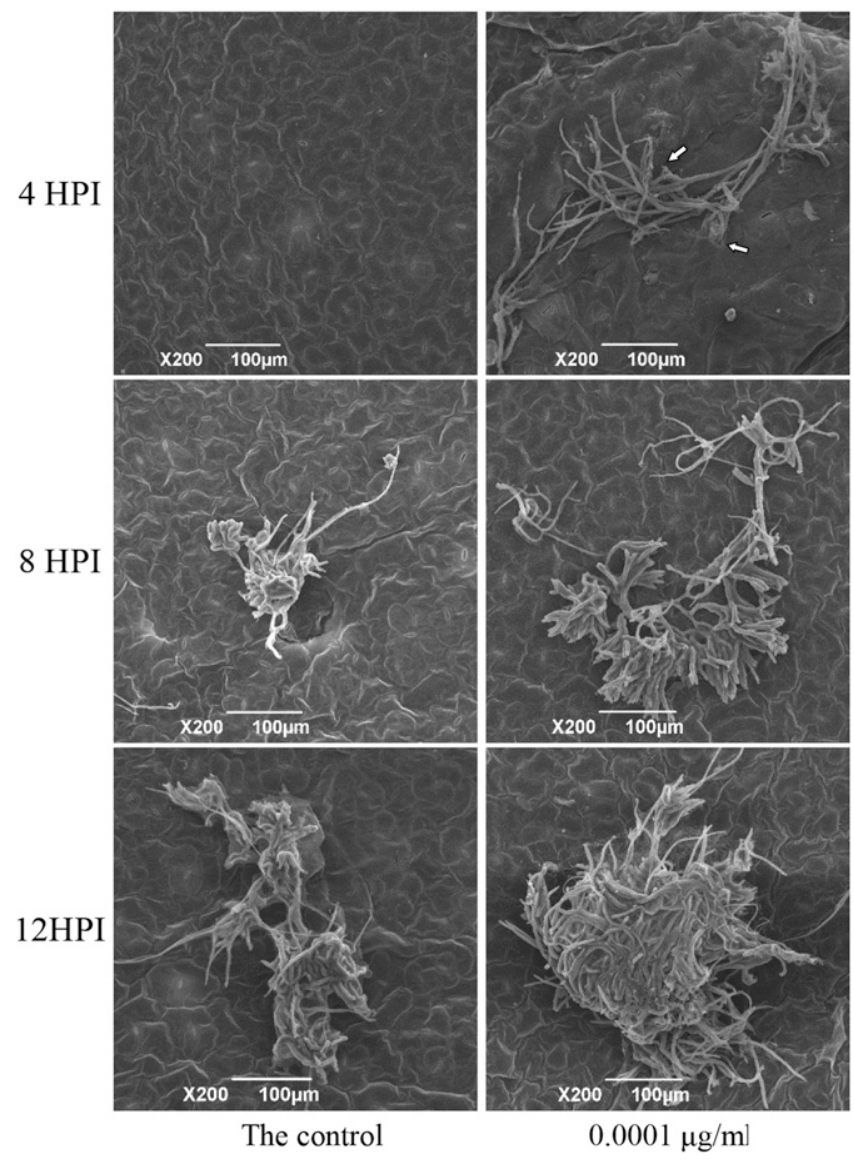

Zhou et al. 2014a). According to mode of action, the three fungicides belong to three different fungicide classes (FRAC 2016), and all three fungicides displayed similar hormetic effects on S. sclerotiorum. It seems very likely that most fungicides at low doses will elicit stimulatory effects on target pathogens. The potential hormetic effects of commercial fungicides are of great concern for effective control of plant pathogens and merit further studies.

The temporal profile of aggressiveness stimulation showed that the earliest sign of stimulation could be detected at 4 HPI with SEM, indicating a direct stimulation mechanism rather than overcompensation to the disruption of homeostasis for hormesis. An overcompensation mechanism can be inferred from the responses of early inhibition and subsequent stimulation, and plenty of hormetic responses caused by overcompensation have been reported in biological and biomedical literature (Calabrese 2001, 2015b). The initial sign of stimulation to aggressiveness could be detected at 4 HPI in the present study, indicating that there was no inhibition on aggressiveness of S. sclerotiorum at the early stage of infections. A study on S. sclerotiorum by Xiao et al. (2014) showed that infection cushions and hyphae penetrating the epidermis of Arabidopsis thaliana leaves could be observed with SEM at 4 HPI. In the present study, no infection cushions or hyphae on rapeseed leaves could be found for the nontreated control at 4 HPI. The discrepancy in infection progress might be attributed to difference in host plant species or ambient humidity. Biochemical mechanisms for the direct stimulation of aggressiveness of S. sclerotiorum are still unclear at the present time. Pharmacological studies demonstrated that direct stimulation may be caused by two distinct subtypes of receptors. One subtype with stimulatory effects has a smaller quantity but a higher affinity for the agonists, whereas the other subtype with inhibitory effects has a larger quantity but a lower affinity for the agonist. Superimposition of

Fig. 4. Scanning electron micrographs showing infection processes of Sclerotinia sclerotiorum isolate HB15 on rapeseed leaves. Leaves sprayed with $0.1 \%$ Triton100 in water were used as the nontreated control. HPI = hours postinoculation. Arrows indicate hyphae penetrating into the epidermis of leaves. 
the opposing effects of the two subtypes of receptors leads to hormetic dose responses (Calabrese 2013, 2015b). Our previous study on hormetic mechanisms has showed that the stimulatory effects of carbendazim on aggressiveness of $S$. sclerotiorum are also caused by a direct stimulation mechanism (Di et al. 2016a ). The knowledge of a direct stimulation mechanism for trifloxystrobin hormetic actions will be conducive to future research on the biochemical mechanisms of fungicide hormesis.

Preconditioning is a specific type of hormesis (Calabrese 2016a,b). This implies that, under field conditions, exposure of pathogens to a fungicide may affect efficacy of subsequent application of the same or related fungicides. The present study demonstrated clearly that pretreating with trifloxystrobin increased tolerance of $S$. sclerotiorum to this fungicide later. Mycelial growth rates on PDA and aggressiveness on rapeseed leaves in the absence of trifloxystrobin did not show significant differences between the pretreatment and nonpretreated control, implying that a prior contact with trifloxystrobin did not directly affect later mycelial growth or aggressiveness. However, in the presence of trifloxystrobin, mycelial growth rates on PDA and aggressiveness on leaves for the pretreatments were significantly higher than for nonpretreatment control, suggesting promoted tolerance to this fungicide. In the present study, tolerance only to trifloxystrobin was assessed. It is highly possible that pretreatment of a pathogen with trifloxystrobin will increase its tolerance to other fungicides, particularly to other members of the QoI class. This warrants further investigations in the future. As with fungicide hormesis, the enhanced tolerance to a fungicide after a prior exposure has profound implications for judicious application of fungicides and should be
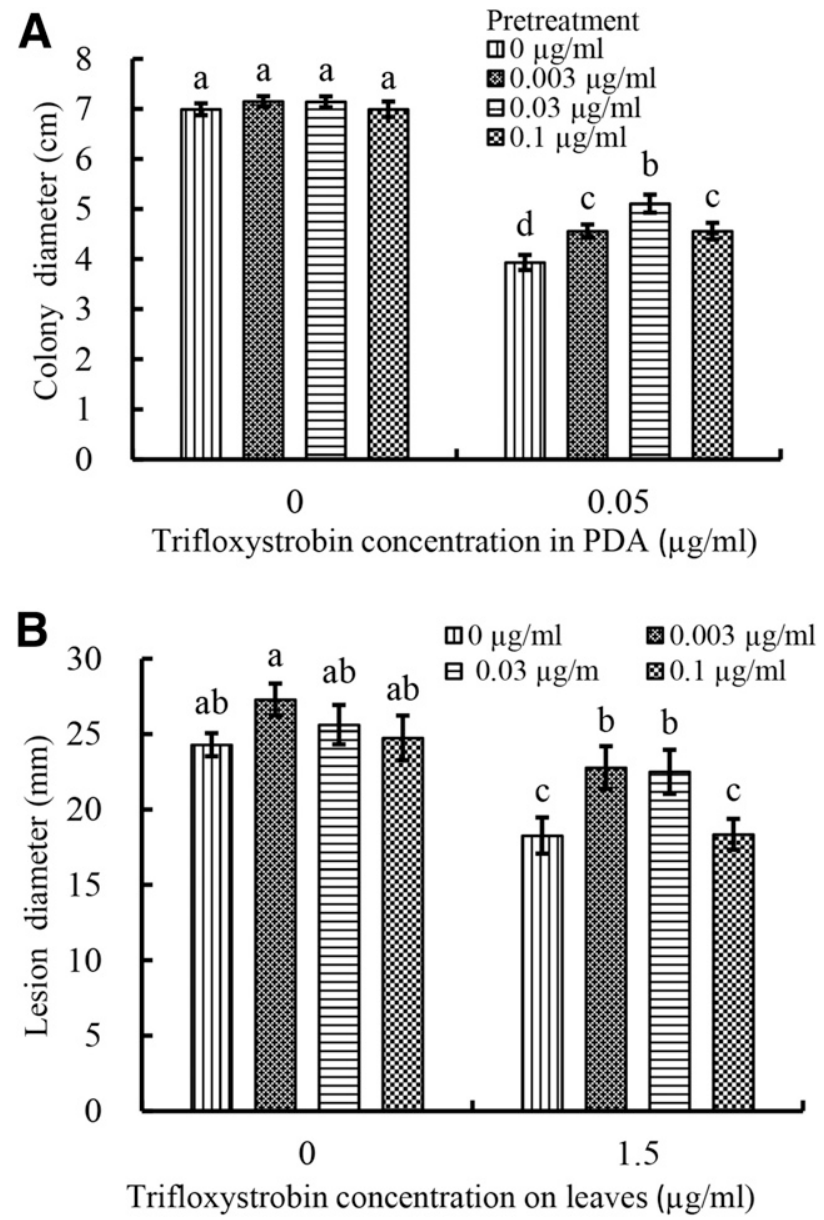

Fig. 5. Effects of pretreatment with trifloxystrobin on A, mycelial growth and B, aggressiveness of Sclerotinia sclerotiorum isolate SX11 in the presence and absence of trifloxystrobin. Data are mean values of 12 replicates. Error bars denote standard error of the mean. Different lowercase letters denote significant differences in lesion diameter among different pretreatments at $\alpha=0.05$. given full attention in developing integrated plant disease management strategies.

\section{Acknowledgments}

This study was supported by National Natural Science Foundation of China (31371964)

\section{Literature Cited}

Baraldi, E., Mari, M., Chierici, E., Pondrelli, M., Bertolini, P., and Pratella, G. C. 2003. Studies on thiabendazole resistance of Penicillium expansum of pears: Pathogenic fitness and genetic characterization. Plant Pathol. 52:362-370

Bartlett, D. W., Clough, J. M., Godwin, J. R., Hall, A., Hamer, M., and ParrDobrzanski, B. 2002. The strobilurin fungicides. Pest Manage. Sci. 58:649-662.

Boland, G. J., and Hall, R. 1994. Index of plant hosts of Sclerotinia sclerotiorum. Can. J. Plant Pathol. 16:93-108.

Bolton, M. C., Thomma, B. P., and Nelson, B. D. 2006. Sclerotinia sclerotiorum (Lib.) de Bary: Biology and molecular traits of a cosmopolitan pathogen. Mol. Plant Pathol. 7:1-16.

Calabrese, E. J. 2001. Overcompensation stimulation: A mechanism for hormetic effects. Crit. Rev. Toxicol. 31:425-470.

Calabrese, E. J. 2013. Hormetic mechanisms. Crit. Rev. Toxicol. 43:580-606.

Calabrese, E. J. 2015a. Hormesis: Principles and applications. Homeopathy 104: 69-82.

Calabrese, E. J. 2015b. Hormesis within a mechanistic context. Homeopathy 104: 90-96.

Calabrese, E. J. 2016a. Preconditioning is hormesis part I: Documentation, doseresponse features and mechanistic foundations. Pharmacol. Res. 110:242264

Calabrese, E. J. 2016b. Preconditioning is hormesis part II: How the conditioning dose mediates protection: Dose optimization within temporal and mechanistic frameworks. Pharmacol. Res. 265-275.

Calabrese, E. J., and Blain, R. B. 2011. The hormesis database: The occurrence of hormetic dose-responses in the toxicological literature. Regul. Toxicol. Pharmacol. 61:73-81.

Di, Y.-L., Lu, X.-M., Zhu, Z.-Q., and Zhu, F.-X. 2016a. Time-course of carbendazim stimulation on pathogenicity of Sclerotinia sclerotiorum indicates a direct stimulation mechanism. Plant Dis. 100:1454-1459.

Di, Y.-L., Zhu, Z.-Q., Lu, X.-M., and Zhu, F.-X. 2015. Pathogenicity stimulation of Sclerotinia sclerotiorum by subtoxic doses of carbendazim. Plant Dis. 99: 1342-1346.

Di, Y.-L., Zhu, Z.-Q., Lu, X.-M., and Zhu, F.-X. 2016b. Baseline sensitivity and efficacy of trifloxystrobin against Sclerotinia sclerotiorum. Crop Prot. 87: 31-36.

Duan, Y. B., Liu, S. M., Ge, C. Y., Feng, X. J., Chen, C. J., and Zhou, M. G. 2012 In vitro inhibition of Sclerotinia sclerotiorum by mixtures of azoxystrobin, SHAM and thiram. Pestic. Biochem. Physiol. 103:101-107.

Firoz, M. J., Xiao, X., Zhu, F. X., Fu, Y. P., Jiang, D. H., Schnabel, G., and Luo, C. X. 2016. Exploring mechanisms of resistance to dimethachlone in Sclerotinia sclerotiorum. Pest Manage. Sci. 72:770-779.

Flores, F. J., and Garzón, C. D. 2013. Detection and assessment of chemical hormesis on the radial growth in vitro of oomycetes and fungal plant pathogens. Dose Response 11:361-373.

FRAC. 2016. FRAC code list 2016: Fungicide sorted by mode of action. Online publication. Fungicide Resistance Action Committee (FRAC). http://www frac.info/docs/default-source/publications/frac-code-list/frac-code-list-2016. pdf?sfvrsn=2

Garzón, C. D., Molineros, J. E., Yanez, J. M., Flores, F. J., Jimenez-Gasco, M. M., and Moorman, G. W. 2011. Sublethal doses of mefenoxam enhance Pythium damping-off of geranium. Plant Dis. 95:1233-1238.

Hoffmann, G. R. 2009. A perspective on the scientific, philosophical, and policy dimensions of hormesis. Dose Response 7:1-51.

Landry, J., Martinez, C., and Rochefort, L. 2011. The use of fungicide Nova to mitigate infection of sphagnum by parasitic fungi in the greenhouse. Botany 89:655-661.

Liang, H. J., Di, Y. L., Li, J. L., You, H., and Zhu, F. X. 2015. Baseline sensitivity of pyraclostrobin and toxicity of SHAM to Sclerotinia sclerotiorum. Plant Dis. 99:267-273.

Ma, H. X., Feng, X. J., Chen, Y., Chen, C. J., and Zhou, M. G. 2009. Occurrence and characterization of dimethachlon insensitivity in Sclerotinia sclerotiorum in Jiangsu Province of China. Plant Dis. 93:36-42.

Margot, P., Huggenberger, F., Amrein, J., and Weiss, B. 1998. CGA279202: A new broad spectrum strobilurin fungicide. Pages 375-382 in: Proc. BCPC Conf. Pests Dis. BCPC, Farnham, Surrey, UK

McCreary, C. M., Depuydt, D., Vyn, R. J., and Gillard, C. L. 2016. Fungicide efficacy of dry bean white mold [Sclerotinia sclerotiorum (Lib.) de Bary, causal organism] and economic analysis at moderate to high disease pressure. Crop Prot. 82:75-81.

Moorman, G. W., and Kim, S. H. 2004. Species of Pythium from greenhouses in Pennsylvania exhibit resistance to propamocarb and mefenoxam. Plant Dis. 88 : 630-632. 
Pan, Y. L., Wang, Z. Y., and Wu, H. Z. 1997. Resistance to carbendazim and stability of the resistance in Sclerotinia sclerotiorum. Jiangsu Agric. Sci. 13: 32-35.

USDA. 2012. National strategic plan for the Sclerotinia research initiative integrated research for disease management in sunflower, canola, dry bean, pea \& lentils and soybean. Online publication. United States Department of Agriculture-Agricultural Research Service. http://www.ars.usda.gov/Research/ docs.htm?docid=20122

Wang, Y., Hou, Y. P., Chen, C. J., and Zhou, M. G. 2014. Detection of resistance in Sclerotinia sclerotiorum to carbendazim and dimethachlon in Jiangsu Province of China. Australas. Plant Pathol. 43:307-312.

Wise, K. A., Bradley, C. A., Pasche, J. S., Gudmestad, N. C., Dugan, F. M., and Chen, W. 2008. Baseline sensitivity of Ascochyta rabiei to azoxystrobin, pyraclostrobin, and boscalid. Plant Dis. 92:295-300.

Xiao, X., Xie, J., Cheng, J., Li, G., Yi, X., Jiang, D., and Fu, Y. 2014. Novel secretory protein Ss-Caf1 of the plant-pathogenic fungus Sclerotinia sclerotiorum is required for host penetration and normal sclerotial development. Mol. Plant-Microbe Interact. 27:40-55.
Xu, C. Y., Hou, Y. P., Wang, J. X., Yang, G. F., Liang, X. Y., and Zhou, M. G. 2014. Activity of a novel strobilurin fungicide benzothiostrobin against Sclerotinia sclerotiorum. Pestic. Biochem. Physiol. 115:32-38.

Xu, D., Pan, Y., Zhang, H., Li, X., Dai, Y., Cao, S., and Gao, Z. 2015. Detection and characterization of carbendazim resistance in Sclerotinia sclerotiorum isolates from oilseed rape in Anhui Province of China. Genet. Mol. Res. 14:16627-16638.

Zhang, L., Wu, M., Li, G., Jiang, D., and Huang, H. 2010. Effect of mitovirus infection on formation of infection cushions and virulence of Botrytis cinerea. Physiol. Mol. Plant Pathol. 75:71-80.

Zhang, S., Panaccione, S. G., and Gallegly, M. E. 1997. Metalaxyl stimulation of growth of isolates of Phytophthora infestans. Mycologia 89:289-292.

Zhou, F., Liang, H. J., Di, Y. L., You, H., and Zhu, F. X. 2014a. Stimulatory effects of sublethal doses of dimethachlon on Sclerotinia sclerotiorum. Plant Dis. 98:1364-1370.

Zhou, F., Zhang, X. L., Li, J. L., and Zhu, F. X. 2014b. Dimethachlon resistance in Sclerotinia sclerotiorum in China. Plant Dis. 98:1221-1226.

Zhu, Z. Q., Zhou, F., Li, J. L., Zhu, F. X., and Ma, H. J. 2016. Carbendazim resistance in field isolates of Sclerotinia sclerotiorum in China and its management. Crop Prot. 81:115-121. 